\title{
A Privacy-Aware Identity Design for Exploring Ubiquitous Collaborative Wisdom
}

\author{
Yuan-Chu Hwang and Soe-Tsyr Yuan \\ Department of Management Information Systems, National Chengchi University, Taiwan \\ No.64, Sec.2, ZhiNan Rd., Wenshan District, Taipei City 11605, Taiwan (R.O.C) \\ \{ychwang, yuans\} @mis.nccu. edu.tw
}

\begin{abstract}
Privacy and security has been considered as the top criterion for the acceptance of e-service adoption. In this paper, we proposed a privacy aware identity protection design that improves the flaw in cheap pseudonym design under the limitation of ambient e-service environment. This design is being applied to a collaborative iTrust platform for ambient e-service, a new scope of mobile ad-hoc e-service. The collaborative iTrust platform highlights the collaborative power from nearby user groups to eliminate potential risk and provide appropriate estimation for trust decision in the ubiquitous environment.
\end{abstract}

Keywords: unlinkability, privacy, collaborative trust.

\section{Introduction to Ambient e-Service}

The notion of ambient e-services is proposed to identify a new scope of ubiquitous e-service, which addresses dynamic collective efforts between mobile users (enabled by Mobile-P2P), dynamic interactions with ambient environments (envisioned by Location-Based Services), moment of value, and low cost service provision [1]. Mobile users can exchange their information wirelessly and proceed highly extensive interactions based on the mobile P2P technology. The collective effort is based on the collaborative interactions of mobile users, which facilitate the low cost service provision. Grounded on location-based service, location information of mobile users can be acquired. Hence, ambient e-service then can provide personal, timely and relevant services to mobile users.

Comparing with the client/server design, an ambient e-service has two major distinguished features. First, it is not possible to effectively attain the collective efforts tailored to the contexts of the users with the client/server architecture. Second, the $\mathrm{P} 2 \mathrm{P}$ design grows the number of connections by a significantly rapid pace especially in an open space.

In order to encourage users to embrace ambient e-service, privacy protection is the vital barrier. Privacy and security are very important concerns today. In ubiquitous eservices environment, who can be trusted? Are the nearby mobile users trustworthy? What do they know about me? These questions underlie the interactions and collaborations between mobile users.

Before we turn to the identity protection design, it will be useful to be aware of the natural limitations of ambient e-service environment. Ambient e-Service is presented 
in a distributed environment, under mobile $\mathrm{P} 2 \mathrm{P}$ ad-hoc network constraints. The mobile device is limited to lightweight computational loading with restricted storage capacity. Moreover, the identities in ambient e-service environment are localized and short-term lived.

In an ambient e-service environment, the mobile peers' composition is dynamic. Users may not stay in the environment for a long time. Mobile peers in such environment form a dynamic-organized structure. Unlike traditional e-commerce environment, users do not have permanent identities. Lack of sustained identification, the trust and reputation data for mobile agents cannot be tracked. The uncertain situation raises the risk for the disclosure of a user's detailed transaction histories to unfamiliar nearby users. How to protect user's private matters under the dynamic environment becomes a major challenge especially when users are encouraged to participate in the collaborative interactions of ambient e-service.

\section{Privacy Aware Identity Protection Design}

Ensuring privacy protection is one of the major incentives to encourage users to embrace the ambient e-service especially when the collective efforts of the e-services were originate from those collaborative interactions between mobile peers. Only when users are assured that their privacy or sensitive data are secured from invasion, the critical mass of the use of the ambient e-service are then unfolded.

In order to design a privacy aware identity protection system for ambient e-service, some critical issues should be considered:

- Unlinkability: Fulfill the untraceable demands for all participants within the ambient e-service environment. Make sure user's identities are not linkable to the real personal identities. Ensure all users within the e-service environment are not able to recognize other's true identity via their involved transaction histories.

- Tolerance of the limitations of ambient e-service environment: The identity protection design should create a fair reputation evaluation mechanism that values user's private matters seriously given the limitations of ambient e-service environment (i.e., capabilities of device computation and storage) while retaining users to participate.

A simple way to achieve unlinkability is pseudonymity, which replaces a user's true identities with a pseudonym used in user interactions. In this paper, a multiple layered pseudonym method is designed for interaction under ambient e-service environment. Before any interactions can be executed, peers require an identity for the service environment. We use the Interaction Pseudonym as an agent identity for the user. It should be noted that a user may possess several different agents for various e-services. An agent's identity is produced according to the service. A user can activate an agent identity or discard a specific identity based on their needs. Even if the user has many agent identities, all identities still share the same global reputation data. When an identity is created, it inherits the concurrent reputation from user's global reputation data. The reputation data for each identity does not exist separately. No matter how many identities belong to the user, he can keep only one global reputation. The diagram (Fig. 1) represents the general design concepts and the relationships of 
three kinds of pseudonyms. Only the Interaction Pseudonyms appear in the interaction environment. Interaction pseudonyms are generating through the same Active Pseudonym but without any linkage relationship. Interaction pseudonyms are cost-free (i.e. cheap pseudonyms); user can generate/discard them freely. However, user can not change their active pseudonym without cost. The algorithm of generating interaction pseudonyms is then shown in Table 1.

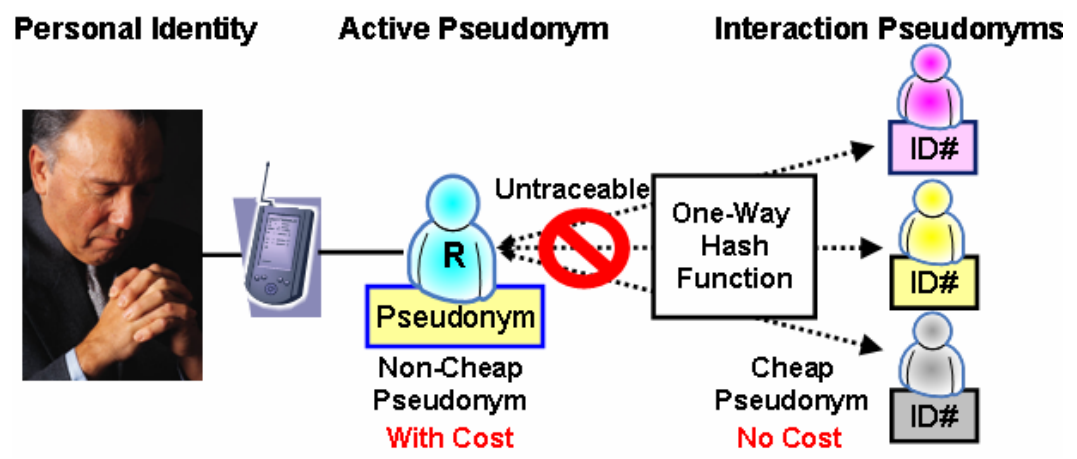

Fig. 1. General idea and relationships between the pseudonyms

The Active Pseudonym represents a pseudonym that entails that the original identity must be responsible for some of the switching cost. As mentioned in section 1, the identities' life-time is short owing to the ambient e-service's nature. However, as addressed in [3], reputation systems generally take on three properties: (1) entities are long-lived; (2) feedback about current interactions is captured and distributed; (3) past feedback guides buyer decisions. Consequently, a conceptual long-lived identity for ambient e-service is indispensable. Opposite to the cheap pseudonyms that allow users to discard cheaply pseudonyms arbitrarily, the non-cheap pseudonym accept user to resume new pseudonyms but charges replacement expense. The non-cheap pseudonym is to encourage users to address themselves on just and honorable behaviors.

The interaction pseudonyms are separated from one another without any linkage to their previous interaction pseudonyms or their personal identities. However, due to the inheritance process, their past behaviors can be ranked by a simplified reputation which prevents the defects of cheap pseudonyms such as whitewashing. (e.g., malicious user may discard their pseudonym, and register a. new pseudonym with a fresh reputation.).

Moreover, a one-way hash function is used to isolate the interaction pseudonyms and the active pseudonym. One-way hash function is an algorithm that turns messages or text into a fixed string of digits, usually for security or data management purposes. The "one way" means that it's nearly impossible to derive the original text from the string. It is also hard to find two strings that would produce the same hash value. As the diagram indicates, even if someone wants to trace a certain person, the only identity can be recognized is his Interaction Pseudonym which is an unlinkable short-term lived identity.

Whenever a user enters the ambient e-service environment, a short-lived interaction pseudonym is created by Anonymizer module. It inherits the briefed reputation 
and assigned role attributes. Because of the time stamp, the interaction pseudonym is localized and temporary valid within the service environment. Once its user leaves the service environment, the interaction pseudonym expires. Even when using the same device, a different interaction pseudonym is assigned to the user when they return to the service environment.

The goals of using our pseudonym design for ambient e-service interactions are three folds: (1).Exclude a unique personal pseudonym for interactions to protect users from possible tracking and profiling. (2). Use multiple interaction pseudonyms to enhance the complexity of identity tracing. (3).Abstract the design of role/relationship pseudonyms for service version selection and delivery. (i.e. Versioning the services by specific types for performance consideration)

Table 1. Interaction Pseudonym Generation Algorithm

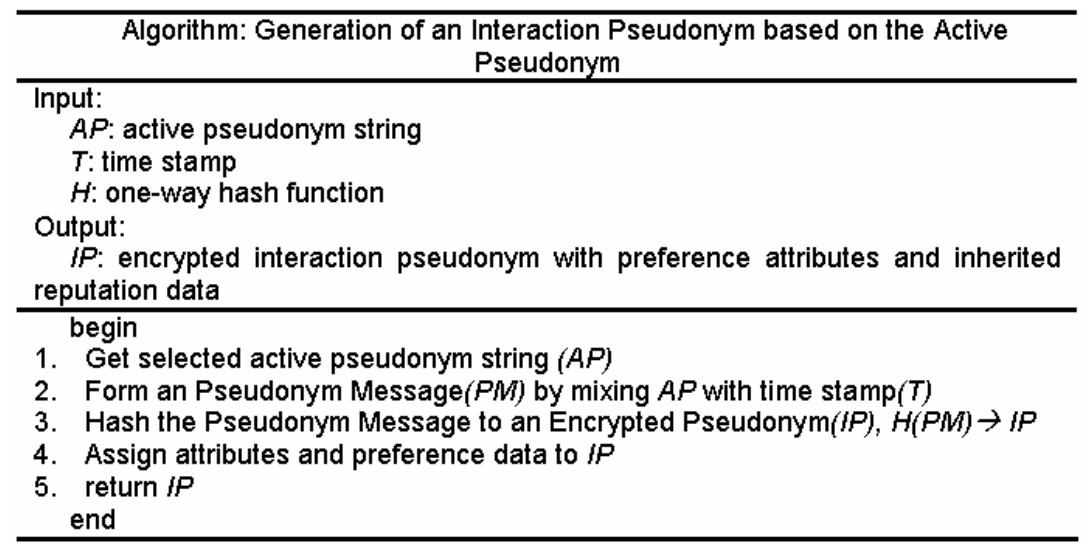

A relevant work of the gradational pseudonym structure was proposed by Pfitzmann and Hansen [2]. They defined the differences between five pseudonym types and classified them into four categories. Their design utilized the classes of pseudonyms for increasing available anonymity and realizing unlinkable transactions. However, their design assumptions were grounded in the concept of centralized and permanent identity management and thus cannot be applied to the ambient e-service environment.

\section{Collaborative iTrust Platform: A Sample Application}

This section exemplifies an application of our identity protection design for ambient e-service so as to the unlinkability of one's personal identity. This application is unfolded with a collaborative iTrust platform that utilizes the privacy aware identity protection design to assure the properties of safety and fairness required for ambient e-Service. The collaborative iTrust platform (shown as Figure 2) includes several function modules. Due to the page limitation, we will elaborate the identity related core modules as follows. 
- Profile Management: In iTrust platform, mobile users can manage their profile settings through a friendly user interface. The profiles includes their preferences and the roles they would like to play, and various attributes such as user's willingness to participate, the will to disclose their interaction experiences, the risk level they can tolerate, and the reliability threshold for determining whether to interact with nearby peers. Once an identity has been generated, those settings will be assigned to the interaction pseudonym automatically.

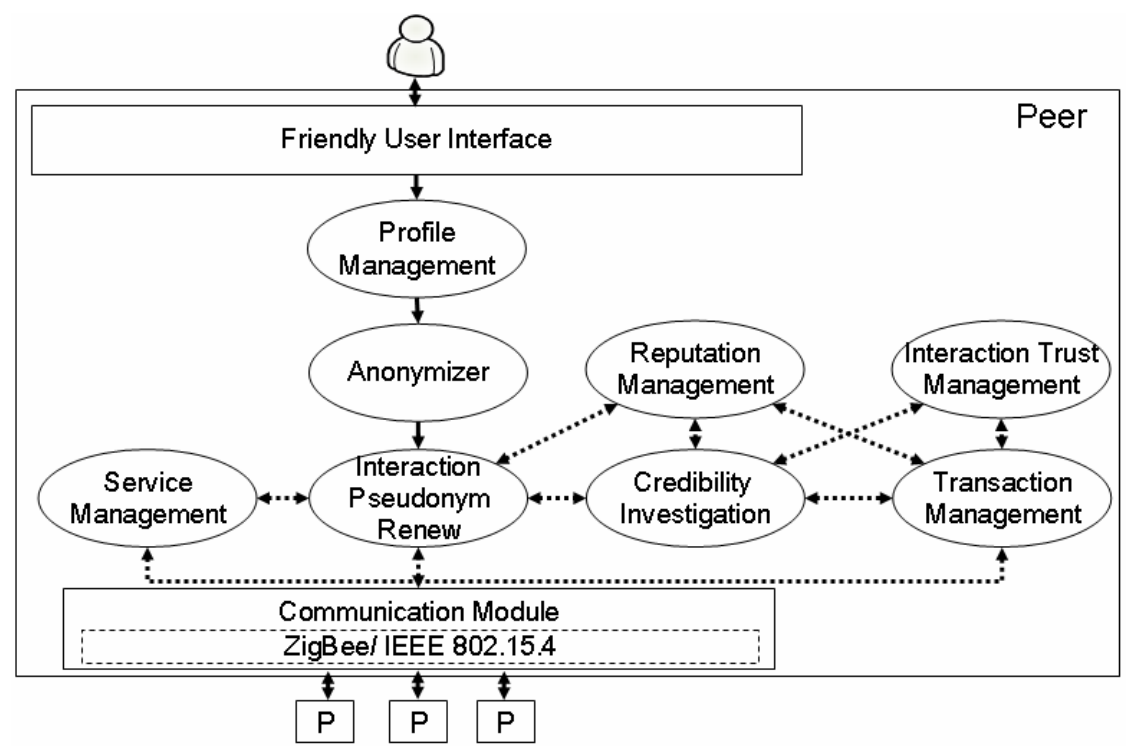

Fig. 2. Macro view of the Collaborative iTrust platform

- Anonymizer: All interactions within the ambient e-service environment are using the "Interaction Pseudonyms" instead of user's real identity or personal pseudonyms. The main function for the Anonymizer is to generate diverse occasional interaction pseudonyms based on their given identity for various kinds of e-services. Those interaction pseudonyms are valid for a short period, and are localized to the corresponding e-service acquired. Because the randomized interaction pseudonyms are not linked to real personal identities and are valid for a limited range, others will be unable to trace their real owner via the interaction pseudonyms. Those interaction pseudonyms are generated by the Anonymizer and will inherit the attribute parameters automatically through the Profile management module. They are able to execute the versioning process and cope with the service management module to reduce irrelevance transmission and improve the efficiency of interaction.

- Interaction Pseudonym Renew: The iTrust design has overcome the problem of the dynamic composition of surrounding peers that may change rapidly. The Interaction Pseudonym Renew module is used to update the list of current nearby users, which exhibits all available nearby peer interaction pseudonyms. Users can interact with peers around themselves through the Communication module. The Interaction 
Pseudonym Renew module is connected with the Reputation Management module, which may immediately update the global reputation of peers so that all devices in range may access it. Each exchange and transmission within the Service Management module, as well as information inquiry when performing creditability investigation, is targeted to those identities obtained by Interaction Pseudonym Renew module.

- Communication Module: The ZigBee based communication module makes use of the security services that are already present in the 802.15.4 security specification. ZigBee infrastructure security includes network access control, integrity of packet routing, and prevention of unauthorized use of packet transport. ZigBee application data security includes message integrity, authentication, freshness, and privacy.[6]

In short, the aforementioned components are tightly coupled with the privacy aware identity protection design for ambient e-service security. However, the capabilities of the collaborative iTrust platform also depend on the functions of additional components (related to the confidential reputation design). The confidential reputation design is intend to modify current public reputation system design and try to separate the detailed transaction history from the reputation data. This separation aims to ward off the bias reputation estimation from vindictive act of users holding up each other or flattering each other to falsify good comments. The confidential reputation design rests on blind-signed scheme that keeping the evaluation targets from being aware of the referee's identity. With this platform design, we expect that users will respond their faithful opinions during their transaction interactions within the ambient eservice environment. Moreover, not only the transaction history can be treated as the data source for trust estimation, the user's direct interaction experience is also considered. Cooperating with the creditability investigation module, the platform provides heterogeneous information based on the user's experiences among one's social network for estimating unfamiliar users.

\section{Significance and Contribution}

Our privacy-aware identity design concept has several beneficial results: (1) Encourage fair transactions that prevent bad users from avoiding punishment. In other words, the design assures bad users cannot regenerate their new identity without cost. (2) For those good users who devote their energies to maintain good reputations, they can accumulate their good reputation and gain some rewards (i.e. get special discount or better chance for transaction) due to the reputation inheritance. This will encourage users to maintain their reputations. With the application of the identity design, the collaborative iTrust platform accordingly is empowered to realize the vision of ambient e-service in terms of the following perspectives:

- Deliberation of short-term lived pseudonyms: Revises existing long term identity design concepts and ensures the unlinkability of identities.

- Distributed data process consideration: Each interaction record within the ambient e-service environment relies on the computational loading and data storage in the mobile device instead of the centralized server database. 
- Lightweight consideration: Different from the existing works of centralized gradational pseudonym design (the quantity of interaction data will be expanded in exponential growth), our design method integrates a user's conceptual role information and relationship information into an abstract public information attached to the user's identity. This attached abstract information can then provide hints to filter out inadequate service information that will reduce unnecessary data transmission. Moreover, the adoption of the time stamp design is exerted to omit those overdue historical interaction data, which will further improve the strength of interaction pseudonym's unlinkability.

- Convenience requirement: Under the versioning scheme, irrelevant service information is filtered out. Only highly correlated services are delivered to the requester. The versioning design reduces communication costs and system loading while improving service efficiency.

A pilot study of user perceived protection involved 38 college students, 24 students male and 14 female. All students had at least two years experience in network communications and understand the potential privacy invasion in an internet environment. The participants were divided into two groups 19 each. Each group was shown a future shopping mall scenario in an ambient e-service environment. We explained that users had to perform shopping activities in a risky environment that contained $50 \%$ honest and 50\% malicious users. The operational interface we showed was the same for both groups, except for the availability of the identity protection design mechanism. Group 1 had a traditional permanent identity, under which users themselves can determine whether to reveal personal information to others. Group 2 was given the multi-layered identity protection design that allows users to use multiple identities for various interactions. Based on the measures for perceived control in [4] and [5], we asked participants about the perceived power of information control and helplessness during privacy protection tasks. $68.4 \%$ of the participants in Group 1 reported that they would prefer to have some advanced protection mechanism for their identities. $84.2 \%$ of Group 2 participants reported that they feel their identities are effectively protected by the identity protection design. For power of information control, no significant difference between two groups $(80.2 \%$ and $78.9 \%)$ was observed. Participants felt that they had the power of information control.

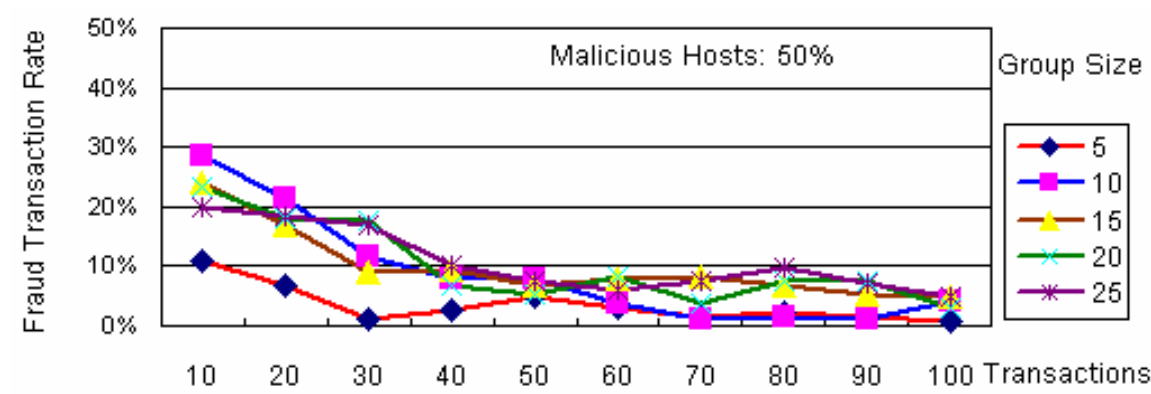

Fig. 3. Evaluation results on decreasing fraud transition rate 
In the ambient e-service environment it is lack of information available for users to estimate which user is trustworthy. This problem is more serious when a new market is opened since the global reputation of each identity is zero and may not satisfy the user's trustworthiness threshold. This will lead to a desolate e-service environment since users may be afraid to transact with unfamiliar users. As the number of transactions increases, more interaction experience is stored in the environment. For the decision quality of the overall collaborative iTrust platform, we develop a prototype system for simulation. Up to 100 transactions were executed by users with different group sizes. In testing the trustworthiness performance, we have considered practical factors such as the population of malicious hosts and good hosts and the available peer numbers for simulating realistic conditions of applications. Figure 3 then shows the evaluation results for collaborative trust measured as successfully decreased fraud transaction rate using our proposed iTrust platform. Even for the risky environment of $50 \%$ malicious hosts, the collaborative trust quickly decreases the presence of malicious transactions. For heterogeneity information sources, the risk level for interact with unfamiliar peer is still significantly decreased when applying the collaborative iTrust platform.

\section{Conclusion and on Going Works}

This paper proposes a privacy aware identity protection design that specifies how the identity protection structure works to resolve the defect of privacy invasion problems for existing e-commerce designs. Furthermore, we also brief a collaborative iTrust platform that exerts the identity design to deliver the visions of ambient e-service with an integrated consideration of trust, reputation and privacy requirements. The identity design aims to encourage the users' participation with the concept of unlinkability (guaranteeing the protection of a user's private matters in the ambient e-service environment). We have implemented the identity design and the device platform, and our on-going works include the evaluation of the design and the platform from different perspectives (trust, reputation, privacy, efficiency, usability, etc.). Preliminary evaluation results indicate the design suits the privacy protection requirement in an ambient e-service environment.

\section{References}

1. Y. Hwang and S. Yuan. A Roadmap for Ambient E-Service: Applications and Embracing Model. International Journal of E-Business Research. 3(1): 51-73. 2007.

2. A. Pfitzmann and M.Hansen. Anonymity, unlinkability, unobservability, pseudonymity, and identity management--- a consolidated proposal for terminology. http://dud.inf.tu-dresden.de/anon-terminology. August, 2005.

3. P. Resnick, R.Zeckhauser, E. Friedman and K. Kuwabara. Reputation systems: Facilitating trust in Internet interactions. Communications of the ACM. 43(12): 45-48. 2000.

4. B. Rohrmann. Empirische Studien zur Entwicklung von Antwortskalen für die sozialwissenschaftliche Forschung. Zeitschrift für Sozialpsychologie. 9: 222-245. 1978.

5. S. Spiekermann. Perceived Control: Scales for Privacy in Ubiquitous Computing Environments. Online Proceedings of the 10th International Conference on User Modeling. Edinburgh, Scotland. July, 2005.

6. ZigBee Org. ZigBee Specification Ver. 1.0, http://www.zigbee.org. 2005. 